\title{
Dispersão da lidocaína administrada por via epidural em cães posicionados em decúbito lateral ou esternal
}

\author{
[Dispersion of lidocaine epidurally administered to dogs positioned on lateral \\ or external decubitus] \\ G.A. Boff ${ }^{1}$, B.M. Naspolini ${ }^{1}$, C.J. de Marco ${ }^{1}$, J.L. Andrades ${ }^{1}$, P. Caye ${ }^{1}$, A.C. Kalb ${ }^{2}$, \\ T.N. Guim ${ }^{3}$, M.I. Gehrcke \\ ${ }^{1}$ Residente - programa de Residência Multiprofissional da Universidade Federal de Pelotas - Pelotas, RS \\ ${ }^{2}$ Aluna de graduação - Universidade Federal de Pelotas - Pelotas, RS \\ ${ }^{3}$ Hospital de Clínicas Veterinárias - Universidade Federal de Pelotas - Pelotas, RS \\ ${ }^{4}$ Universidade Federal de Pelotas - Pelotas, RS
}

\begin{abstract}
RESUMO
O objetivo do estudo foi verificar clinicamente a dispersão da lidocaína no espaço epidural de cães posicionados em diferentes decúbitos. Foram utilizados 16 cães, com peso médio de 17,5 quilogramas. Esses foram tranquilizados com acepromazina, anestesiados com propofol e alocados em dois grupos, conforme o decúbito de posicionamento: decúbito esternal (GE) e decúbito lateral direito (GLD). Ambos os grupos receberam lidocaína a $2 \%$, no volume de $0,25 \mathrm{~mL} / \mathrm{kg}$, e permaneceram no mesmo decúbito por 20 minutos. Em seguida, avaliou-se o bloqueio dos membros pélvicos e a extensão do bloqueio, a partir da sétima vértebra lombar, por meio de pinçamento interdigital e do panículo paravertebral. Foi, então, realizada cirurgia de orquiectomia. Após tal procedimento, avaliou-se o tempo total de bloqueio dos membros pélvicos. Todos os cães apresentaram bloqueio bilateral, sem diferenças quanto à extensão cranial entre os grupos, sendo a mediana de 7,5 (1-14) vértebras para GE e de 4 (1-14) para GLD. O tempo de bloqueio dos membros direito e esquerdo foi de $123 \pm 26$ e $130 \pm 20$ minutos, para GE, e de $120 \pm 21$ e $121 \pm 20$ minutos, para GLD, sem diferenças entre os grupos ou entre os membros. Conclui-se que o decúbito não interfere na distribuição da lidocaína administrada por via epidural.
\end{abstract}

Palavras-chave: anestesia local, peridural, canina

\section{ABSTRACT}

The aim of this study was to clinically verify the dispersion of lidocaine in the epidural space of dogs placed in different positions. Sixteen dogs with an average weight of 17.5 kilograms were used. These were tranquilized with acepromazine, anesthetized with propofol and allocated to two groups: sternal decubitus (GE) and right lateral decubitus (GLD). Both groups received $2 \%$ of lidocaine in the volume of $0.25 \mathrm{~mL} / \mathrm{kg}$ and remained in the same position for 20 minutes. The blocking of the pelvic limbs and the extension of it from the seventh lumbar vertebra were evaluated by means of interdigital and paravertebral panniculus clamping. Orchiectomy surgery was then performed. Afterwards, the total blocking time of the pelvic limbs was evaluated. All dogs presented bilateral blocking, with no differences in cranial extension between groups, with a median of 7.5 (1-14) vertebrae for GE and 4 (1-14) for GLD. The blocking time of the right and left limbs were $123 \pm 26$ and $130 \pm 20$ minutes for GE, and $120 \pm 21$ and $121 \pm 20$ minutes for GLD with no difference between groups or between limbs. It is concluded that the decubitus does not interfere with the epidural lidocaine distribution.

Keywords: local anesthetic, peridural, canine

\section{INTRODUÇÃO}

A anestesia epidural lombossacral é uma técnica de anestesia local amplamente utilizada para realização de cirurgias no abdômen retroumbilical, no períneo e nos membros pélvicos, em cães e gatos (Jones, 2001; Valverde,
2008; Steagall et al., 2017; Ferreira, 2018; GarciaPereira, 2018). Nos mamíferos, os fatores que afetam a distribuição epidural são individuais, como a idade, a anatomia e o escore corporal e técnico, relacionados com a viscosidade do fluido, volume administrado, velocidade de administração e posicionamento (Visser et al., 
2008). Em humanos (Hodgkinson e Husain, 1980; Merry et al., 1983), a realização da técnica com o paciente sentado ou em decúbito dorsal interfere na dispersão, obedece às forças gravitacionais e tende ao acúmulo de anestésico nas regiões inferiores. Com base nessa premissa, quando há intenção de se realizar um bloqueio unilateral, a manobra de deixar o sítio cirúrgico voltado para baixo, após realização da anestesia epidural, é recomendada na literatura veterinária (Jones, 2001; Valverde, 2008; Steagall et al., 2017).

Em cães, a administração de azul de metileno pela via epidural é afetada pelo decúbito (Gorgi et al., 2006). Os autores concluíram que a permanência em decúbito lateral direito por 40 minutos, após a administração epidural, leva a um maior acúmulo do lado direito e a uma dispersão mais cranial. Contudo, a avaliação da dispersão em cadáveres é limitada, por não representar um sistema orgânico vivo, com um sistema hemodinâmico ativo (Valverde, 2008). Ainda, as características moleculares de peso e estrutura do azul de metileno diferem das características dos anestésicos locais e de outros medicamentos administrados por via epidural.

Steagall e colaboradores (2017) relataram que, na experiência dos autores, a permanência do sítio cirúrgico voltado para baixo, no período de preparação da cirurgia, é válida para promover maior dispersão epidural nas fibras nervosas da região a ser operada. Entretanto, o embasamento para essas afirmações é de conteúdo experimental, com visualização direta com azul de metileno e não clínico, com anestésicos locais e procedimentos cirúrgicos. Por fim, revisões mais recentes acerca da anestesia epidural não discutem a interferência do posicionamento na dispersão após a administração epidural (Ferreira, 2018; Garcia-Pereira, 2018), embora muitos profissionais adotem essa medida. O objetivo do presente estudo foi avaliar clinicamente a dispersão da lidocaína pela observação do bloqueio sensitivo dos membros, da extensão cranial e do tempo de bloqueio, em cães que permaneceram em decúbito esternal ou lateral direito, logo após bloqueio epidural lombossacral com lidocaína, e foram submetidos à cirurgia eletiva de orquiectomia.

\section{MATERIAL E MÉTODOS}

O estudo foi submetido ao Comitê de Ética em Experimentação Animal da Universidade Federal de Pelotas e foi aprovado segundo protocolo $n .^{\circ}$ 0499-18. Foram utilizados 16 cães machos hígidos, pesando em média 17,5 \pm 5 quilogramas e com idade média de $5 \pm 1,3$ anos, encaminhados para orquiectomia eletiva. Todos os animais foram classificados como ASA I, após exames físico e laboratorial.

Previamente ao procedimento, os cães foram submetidos a jejum alimentar de oito horas e hídrico de duas. Os animais foram tranquilizados com acepromazina (Apromazin, maleato de acepromazina, Syntec, Brasil), na dose de $0,05 \mathrm{mg} / \mathrm{kg}$, pela via intramuscular. Decorridos 20 minutos da administração, foi realizada tricotomia para acesso venoso, sítio cirúrgico e região lombossacral. Em seguida, os cães foram encaminhados para o centro cirúrgico, onde foi realizado acesso venoso pela veia cefálica e iniciada fluidoterapia com ringer com lactato na taxa de $5 \mathrm{~mL} / \mathrm{kg} / \mathrm{h}$.

Foi realizada a administração de propofol (Provive, propofol, Claris, Brasil), na dose de $2 \mathrm{mg} / \mathrm{kg}$, por via intravenosa, seguida de doses adicionais de $1 \mathrm{mg} / \mathrm{kg}$ quando necessário, até se atingir plano superficial de anestesia, suficiente para se realizar a punção do espaço epidural. Os cães, então, foram aleatoriamente distribuídos em dois grupos, conforme o decúbito de execução da técnica de anestesia epidural: decúbito esternal (GE), com os membros posteriores tracionados cranialmente, em posição de esfinge, e lateral direito (GLD), com os membros tracionados na direção cranial.

Ato contínuo, foi realizada antissepsia cirúrgica na região lombossacral e realizada punção epidural, por meio de agulha de Tuohy $18 \mathrm{G}$, por onde foi administrada lidocaína $2 \%$ sem vasoconstritor (Hypocaína, cloridrato de lidocaína, Hypofarma, Brasil), na dose de $0,25 \mathrm{~mL} / \mathrm{kg}$, na taxa de $6 \mathrm{~mL} / \mathrm{min}$, sempre pelo mesmo anestesista. A confirmação do acesso ao espaço epidural foi realizada pela sensação de "pop", ao atravessar o ligamentum flavum, pela aspiração de uma gota de anestésico depositada no canhão da agulha ou pela perda da resistência à administração.

Após administração epidural, os cães permaneceram nas posições esternal (GE) e lateral 
direita (GLD), conforme o grupo, por 20 minutos, e, quando necessário, foi realizada contenção física. Passados os 20 minutos, os cães do grupo esternal foram posicionados na posição lateral direita e, nesse momento, um avaliador cego às posições de administração realizou a avaliação sensitiva do bloqueio, com o uso de pinça hemostática fechada até a primeira serrilha, na região interdigital de ambos os membros pélvicos. O reflexo foi considerado positivo quando houve retirada ou contração do membro. Após avaliação sensitiva dos membros, a extensão cranial foi verificada com pinçamento da pele, na altura de cada processo vertebral, a partir da sétima vértebra lombar, até que houvesse reflexo do panículo presente.

Ao final da avaliação sensitiva, os cães foram induzidos à anestesia geral com propofol, na dose de $5 \mathrm{mg} / \mathrm{kg}$, por via intravenosa, intubados com sonda traqueal de tamanho adequado e posicionados em decúbito dorsal. Com o paciente conectado ao sistema de anestesia circular valvular, a manutenção anestésica foi realizada com isoflurano (Isoflurano, isoflurano, BioChimico, Brasil), na vaporização de 1,2 a $1,5 \mathrm{~V} \%$, por meio de analisador de gases e diluído em oxigênio $100 \%$.

Durante o procedimento, foram avaliados os parâmetros de pressão arterial sistólica (PAS), por meio de Doppler vascular e manômetro aneroide; frequência cardíaca (FC), com uso de eletrocardiograma na derivação II; saturação parcial de oxigênio periférico $\left(\mathrm{SPO}_{2}\right)$, por sensor posicionado na língua; frequência respiratória (FR); pressão e concentração final expirada de $\mathrm{CO}_{2}\left(\mathrm{EtCO}_{2}\right)$ e isoflurano, por meio de monitor multiparamétrico com analisador de gases. Os parâmetros foram monitorados previamente ao início da cirurgia (M0), logo após a incisão cirúrgica (M1), ao pinçar o primeiro plexo testicular (M2), após pinçar o segundo plexo (M3), e, a partir deste momento, a cada cinco minutos até o final da cirurgia.

Durante as mensurações, caso o paciente apresentasse aumento da pressão arterial acima de $20 \%$ do valor anterior, foi considerado estímulo nociceptivo presente, então, foi administrado fentanil (Fentanest, citrato de fentanila, Cristália, Brasil), na dose de $3 \mu \mathrm{g} / \mathrm{kg}$, por via intravenosa. Se a frequência cardíaca diminuísse abaixo de $60 \mathrm{bpm}$, seria administrada atropina (Pasmodex, sulfato de atropina, Isofarma, Brasil), na dose de $0,022 \mathrm{mg} / \mathrm{kg}$, e, se a pressão diminuísse abaixo de $90 \mathrm{mmHg}$, seria administrado bolus de solução fisiológica de $\mathrm{NaCl}^{-} 0,9 \%$, em $90 \mathrm{~mL} / \mathrm{kg} / \mathrm{h}$, por cinco minutos, por duas tentativas, seguida da administração de efedrina (Efedrin, sulfato de efedrina, Cristália, Brasil), na dose de $0,1 \mathrm{mg} / \mathrm{kg}$, se necessário. Após o término do procedimento, no período pós-operatório, o tempo de bloqueio foi avaliado a cada 10 minutos, por compressão interdigital, até restabelecimento do reflexo sensitivo.

Para análise estatística, foi utilizado o programa GraphPad Prism 7. Foi realizado teste de normalidade de Shapiro-Willk. As variáveis em cada grupo foram avaliadas pelo teste ANOVA e pós-teste de Dunnett, nas variáveis paramétricas, ou pelo teste Kruskal-Wallis e pós-teste de Dunn, nas não paramétricas. A comparação entre os grupos foi realizada pelos testes $\mathrm{t}$, nas variáveis paramétricas, e de Mann-Whitney, nas não paramétricas. Todas as análises foram realizadas com nível de significância de 5\%.

\section{RESULTADOS}

A confirmação da efetividade do bloqueio epidural se deu por meio da ausência de resposta ao pinçamento, do relaxamento de esfíncter anal e da ausência de mobilidade. Os bloqueios dos membros foram bilaterais em todos os cães de ambos os grupos. A extensão cranial observada pelo reflexo do panículo, em ambos os grupos, variou entre o primeiro e o $14^{\circ}$ processo vertebral pinçado, sem diferença entre os grupos. O tempo de bloqueio também não diferiu entre os grupos nem entre os membros de cada grupo, conforme descrito na Tab. 1.

As variáveis paramétricas de frequência cardíaca (FC), frequência respiratória (FR), pressão parcial de dióxido de carbono expirado (ETCO2), saturação de oxigênio no sangue periférico (SatO2) e pressão arterial sistólica (PAS) estão apresentadas na Tab. 2. As FC e FR diminuíram entre a avaliação antes da cirurgia (M0) e as avaliações que se seguiram à cirurgia (M3 e M4), em ambos os grupos. Ainda, a FC aumentou no M2 em relação ao M0, no grupo GLD. Também foi observado aumento nos valores da PAS, entre os momentos M0 e M2, no grupo GE. Por fim, a pressão de $\mathrm{ETCO}_{2}$ apresentou valores maiores no momento M3 em relação ao M0, no grupo GE. 
Tabela 1. Extensão cranial em número de processos vertebrais pinçados até a presença do reflexo do panículo, descritos em mediana (máxima e mínima), do bloqueio dos membros (número de animais/bloqueio positivo) e do tempo de bloqueio em minutos, apresentados em média e desvio-padrão, dos membros direito e esquerdo. Parâmetros avaliados em cães distribuídos em grupos esternal (GE) e lateral direito (GLD), avaliados 20 minutos após a realização da anestesia epidural e a cada 10 minutos após término da cirurgia de orquiectomia

\begin{tabular}{lllc}
\multicolumn{1}{c}{ Avaliação } & Grupos & Membros & Valores \\
\hline \multirow{2}{*}{ Extensão cranial } & GE & & $7,5(1-14)$ \\
& GLD & & $4(1-14)$ \\
& & Direitos & $8 / 8$ \\
Bloqueio dos membros & GE & Esquerdos & $8 / 8$ \\
& & Direitos & $8 / 8$ \\
& GLD & Esquerdos & $8 / 8$ \\
& & Direitos & $123 \pm 26$ \\
Tempo de bloqueio dos membros & GE & Esquerdos & $130 \pm 20$ \\
& \multirow{2}{*}{ GLD } & Direitos & $120 \pm 21$ \\
& & Esquerdos & $121 \pm 20$ \\
\hline
\end{tabular}

Tabela 2. Variáveis de frequência cardíaca (FC), frequência respiratória (FR), pressão parcial de gás carbônico ao final da expiração $\left(\mathrm{ETCO}_{2}\right)$, saturação de oxigênio no sangue periférico $\left(\mathrm{SatO}_{2}\right)$ e pressão arterial sistólica (PAS). Prévio à incisão da pele (M0) e ao longo do procedimento cirúrgico, nos momentos de incisão da pele (M1), pinçamento do primeiro plexo testicular (M2), pinçamento do segundo plexo testicular (M3) e síntese de pele (M4). Parâmetros avaliados durante o procedimento cirúrgico de orquiectomia em cães. Previamente, os cães foram distribuídos em dois grupos: esternal (GE) e lateral direito (GLD), para realização da anestesia epidural

\begin{tabular}{|c|c|c|c|c|c|c|}
\hline Parâmetro & Grupo & M0 & M1 & M2 & M3 & M4 \\
\hline \multirow{2}{*}{$\mathrm{FC}$} & GE & $105 \pm 18$ & $104 \pm 11$ & $117 \pm 25$ & $79 \pm 16^{*}$ & $79 \pm 18^{*}$ \\
\hline & GLD & $104+22$ & $105 \pm 14$ & $125 \pm 18^{*}$ & $77 \pm 11^{*}$ & $76 \pm 12^{*}$ \\
\hline \multirow{2}{*}{ FR } & GE & $12 \pm 3$ & $11 \pm 2$ & $10 \pm 2$ & $6 \pm 2^{*}$ & $7 \pm 2^{*}$ \\
\hline & GLD & $11 \pm 3$ & $12 \pm 4$ & $11 \pm 3$ & $6 \pm 1^{*}$ & $6 \pm 2^{*}$ \\
\hline \multirow{2}{*}{ PAS } & $\mathrm{GE}$ & $105 \pm 9$ & $101 \pm 25$ & $134 \pm 15^{*}$ & $110 \pm 15$ & $110 \pm 22$ \\
\hline & GLD & $110 \pm 21$ & $109 \pm 20$ & $141 \pm 27$ & $116 \pm 25$ & $116 \pm 35$ \\
\hline \multirow{2}{*}{$\mathrm{SatO}_{2}$} & GE & $99 \pm 1$ & $99 \pm 1$ & $100 \pm 1$ & $99 \pm 1$ & $100 \pm 1$ \\
\hline & GLD & $99 \pm 1$ & $99 \pm 1$ & $100 \pm 1$ & $99 \pm 1$ & $99 \pm 1$ \\
\hline \multirow{2}{*}{$\mathrm{ETCO}_{2}$} & $\mathrm{GE}$ & $34 \pm 4$ & $35 \pm 4$ & $35 \pm 3$ & $38 \pm 2^{*}$ & $38 \pm 4$ \\
\hline & GLD & $32 \pm 3$ & $33 \pm 3$ & $33 \pm 2$ & $40 \pm 8$ & $37 \pm 4$ \\
\hline
\end{tabular}

* Diferença de $\mathrm{P}<0,05$ entre os momentos cirúrgicos e o M0.

Nos oito cães de ambos os grupos, foi necessário um bolus de fentanil, por aumento do valor de pressão arterial sistólica acima de $20 \%$ da mensuração anterior, correspondente ao momento de pinçar o primeiro plexo testicular (M2). Foi realizado bolus de atropina em um cão de cada grupo, ambos os grupos correspondentes a momentos que se seguiram à administração do fentanil (M3 e M4). Em um cão, do grupo GE, também nos momentos que se seguiram à administração do fentanil, fez-se necessária a administração de bolus de fluido, para restabelecer a pressão arterial sistólica acima de $90 \mathrm{mmHg}$.

\section{DISCUSSÃO}

Em todos os animais de ambos os grupos, houve a certeza do correto posicionamento da agulha, devido ao teste de perda da resistência e à presença do bloqueio sensitivo dos membros. Contudo, o teste da gota pendente só foi presente em alguns animais do grupo esternal e em nenhum do grupo lateral direito, o que, somado à posição corporal, torna a realização da técnica em decúbito lateral mais difícil. A razão de o teste ser negativo em pacientes em decúbito lateral é explicada em estudos em cães, suínos e bovinos (Bengis e Guyton, 1977; Nystrom et al., 1998; 
Lee et al., 2002). Com o posicionamento em decúbito lateral, a pressão abdominal aumenta o fluxo sanguíneo e, consequentemente, provoca vasodilatação do plexo vascular lombar, o que aumenta a pressão no espaço epidural e interfere na absorção da gota depositada no canhão da agulha (Naganobu e Hagio, 2007).

No presente estudo, houve dispersão cranial do anestésico local, sem diferença entre os grupos. Gorgi e colaboradores (2006) verificaram que a injeção de azul de metileno, no espaço epidural de cães, apresenta dispersão mais cranial e lateralizada em cães mantidos em decúbito lateral durante 40 minutos, diferentemente dos resultados do presente estudo. Contudo, os resultados dos autores são de um estudo pósmorte, que não mimetiza as características de um organismo vivo, em que há circulação sanguínea e ocorre absorção dos fármacos (Valverde, 2008). Também cabe ressaltar as diferenças químicas da lidocaína e do azul de metileno, que podem afetar a distribuição de ambos. Sobre a dispersão máxima no espaço epidural, acredita-se que o tempo de 20 minutos de espera para aferição do bloqueio foi correto, já que corrobora o tempo do ápice de dispersão de contraste radiográfico e de bupivacaína no espaço epidural de cães (Freire et al., 2010; Zhang et al., 2013). Assim, acredita-se que um maior tempo de espera não afetaria a lateralidade ou a dispersão cranial do bloqueio.

Nas condições do presente estudo, não houve bloqueio unilateral, e os tempos de bloqueio não diferiram entre os grupos ou entre os membros de cada grupo, ficando próximos dos valores já relatados em outros estudos (Ishiy et al., Gasparini et al., 2007; Cassu et al., 2008). Isso demonstra que não houve distribuição unilateral da lidocaína nos pacientes do GLD, o que vai de encontro às recomendações da literatura para posicionamento do paciente em decúbito dependente da região cirúrgica (Jones, 2001; Gorgi et al., 2006; Valverde, 2008; Steagall et al., 2017).

Seres humanos submetidos à anestesia com cateteres posicionados no espaço epidural lateral, por meio da rotação da agulha no momento de implantação do cateter, apresentaram maior incidência de anestesias unilaterais, menor consumo de anestésico local, maior tempo de analgesia sem resgate, menor tempo sem caminhar e menor incidência de retenção urinária no período pós-cirúrgico (Borgui et al., 2004; Sen et al., 2012). As razões que levam a encontrar resultados diferentes em cães estão relacionadas com a metodologia. No presente estudo, a técnica de anestesia epidural era realizada com agulha em punção única e em velocidade constante.

Assim, considerando que o espaço epidural é cilíndrico, a administração em bolus, no centro do cilindro, promove dispersão circunferencial total e progressão cranial uniforme. Outra possibilidade é a de que, caso a administração de volumes menores de anestésico fosse realizada de forma lenta, nos pacientes em decúbito lateral, poderia haver uma distribuição mais lateral. Contudo, são necessários outros estudos para afirmar tal teoria.

Em relação à variabilidade da extensão do bloqueio, esta pode ser devido a fatores individuais de cada paciente e é relatada em pesquisas de distribuição. Estudos de dispersão com corante concluíram que a distribuição é desuniforme e pode sofrer interferência de barreiras de gordura, tendendo a seguir, na maioria das vezes, o caminho da vascularização presente no espaço epidural (Lee et al., 2001; Hogan, 2002; Gorgi et al., 2006; Son et al., 2011). Por meio de avaliação clínica, Freire et al. (2010) verificaram o bloqueio sensorial de $5 \pm 3,3$ dermátomos após a administração de $0,2 \mathrm{~mL} / \mathrm{kg}$ de bupivacaína por via epidural. Também com bupivacaína, no mesmo volume e via, Son e colaboradores (2014) observaram bloqueio de 3,5 (0-20) dermátomos e grande variação individual. Já Gasparini et al. (2007) administraram, por via epidural, lidocaína, no volume de $0,25 \mathrm{~mL} / \mathrm{kg}$, e alcançaram 2,4 dermátomos bloqueados. Esses achados ratificam a variabilidade da progressão de acordo com os fármacos e métodos empregados na avaliação.

$\mathrm{Na}$ variação de valores dos sinais vitais, as diferenças de FC, FR, ETCO 2 e PAS, em ambos os grupos, são entre o momento M0 e os momentos M3 e M4. Essas alterações foram notadas especialmente após o resgate analgésico com fentanil. Estudos relacionaram a administração de fentanil com a diminuição das frequências cardíaca (Steagall et al., 2006) e respiratória em cães (Bailey et al., 1990; Salmenpera et al., 1994). Apesar das diferenças ao longo do tempo, os parâmetros de FC, FR, $\mathrm{ETCO}_{2}, \mathrm{SatO}_{2}$ e PAS se mantiveram em valores 
aceitáveis para cães submetidos à anestesia epidural com lidocaína, anestesia com isoflurano e submetidos à castração (Ishiy et al., 2002). Não houve diferença entre os efeitos adversos de bradicardia e hipotensão, entre os grupos, apesar de um cão de cada grupo apresentar bradicardia, e um do grupo GE hipotensão. Esses fenômenos foram observados após a administração do fentanil.

O sistema nervoso autônomo é responsável pela informação nociceptiva visceral que ascende ao sistema nervoso central, mediada pelas fibras nociceptivas do tipo C (Nesse Gebhart, 1990). O aumento de pressão arterial sistólica acima de $20 \%$ dos valores anteriores, no momento em que o primeiro plexo testicular foi pinçado, sugere uma resposta nociceptiva ao estímulo. A enervação somática testicular que emerge dos ramos vertebrais lombares é descrita por Rocha $e t$ al. (1990), no entanto, no presente estudo, esses ramos estavam bloqueados em alguns dos cães de ambos os grupos. Porém, os testículos também recebem fibras nervosas autonômicas, oriundas de nervos do plexo aórtico abdominal (Ghoshal, 1986), o que pode explicar a percepção nociceptiva presente no momento de pinçar o plexo testicular. Esse fenômeno também foi descrito na cirurgia de ovário-histerectomia, em cadelas que receberam lidocaína, na mesma dose, via e volume do presente estudo (Ishiy et al., 2002). Cabe ressaltar que, como a altura de bloqueio foi testada por meio de reflexo do panículo, esta pode apresentar variação na altura do bloqueio.

\section{CONCLUSÃO}

Conclui-se que não há diferenças na lateralização, na altura e no tempo de bloqueio anestésico da lidocaína, administrada por via epidural lombossacral, em cães em decúbito lateral, quando comparado ao decúbito esternal. Assim, não há necessidade de lateralização dos pacientes ou posicionamento prévio do paciente em decúbito dependente em caso de cirurgias unilaterais.

\section{REFERÊNCIAS}

BAILEY, P.L.; PACE, N.L.; ASHBURN, M.A. et $a l$. Frequent hypoxemia and apnea after sedation with midazolam and fentanyl. Anesthesiology, v.73, p.826-830, 1990.
BENGIS, R.G.; GUYTON, A.C. Some pressure and fluid dynamic characteristics of the canine epidural space. Am. J. Phys., v.232, p.255-259, 1977.

BORGUI, B.; AGNOLETTI, V.; RICCI, A. et al. Prospective, randomized evaluation of the effects of epidural needle rotation on the distribution of epidural block. Aneasth. Analg., v.98, p.14731478, 2004.

CASSU, R.N.; STEVANIN, H.; KANASHIRO, C. et al. Anestesia epidural com lidocaína isolada e associada ao fentanil para realização de ováriossalpingohisterectomia em cadelas. Arq. Bras. Med. Vet. Zootec., v.60, p.825-831, 2008.

FERREIRA, J.P. Epidural anaesthesia-analgesia in the dog and cat: considerations, technique and complications. Comp. Anim. v.23, p.628-636, 2018.

FREIRE, C.D.; TORRES, M.L.A.; FANTONI, D.T. et al. Bupivacaine $0.25 \%$ and methylene blue spread with epidural anesthesia in dog. Vet. Aneasth. Analg., v.37, p.63-69, 2010.

GARCIA-PEREIRA, F. Epidural anesthesia and analgesia in small animal practice: an update. Vet. J., v.242, p.24-32, 2018.

GASPARINI, S.S.; LUNA, S.T.L.; CASSU, R.N. et al. Anestesia epidural com ropivacaína, lidocaína ou associação de lidocaína e xilazina em cães. Efeitos cardiorrespiratório e analgésico. Cienc. Rural, v.37, p.418-424, 2007.

GHOSHAL, N.G. Sistema nervoso. In: GETTY R.; SISSON S.; GROSSMAN, J.D. Sisson/Grossman: anatomia dos animais domésticos. 5.ed. Rio de Janeiro: Guanabara Koogan, 1986. cap.57, p.1569-1617.

GORGI, A.A.; HOFMEISTER, E.H.; HIGGINBOTHAM, M.J. et al. Effect of body position on cranial migration of epidurally injected methylene blue in recumbent dogs. Am. J. Vet. Res., v.67, p.219-221, 2006.

HODGKINSON, R.; HUSAIN, F.J. Obesity and the cephalad spread of analgesia following epidural administration of bupivacaine for cesarean section. Aneasth. Analg. v.59, p.89-92, 1980.

HOGAN; Q. Distribution of solution in the epidural space: examination by cryomicrotome section. reg. Anesth. Pain Med., v.27, p.150-156, 2002. 
ISHIY, H.M.; LUNA, S.P.L.; GONÇALVES, R.C. et al. Uso da lidocaína isolada ou associada à quetamina ou ao butorfanol, em anestesia epidural em cadelas submetidas à ovariosalpingohisterectomia. Rev. Bras. Ciên. Vet., v.9, p.134-136, 2002.

JONES, R.S. Epidural analgesia in the dog and cat. Vet. J., v.161, p.123-131, 2001.

LEE, I.; SOEHARTONO, R.H.; YAMAGISHI, N. et al. Distribution of new methylene blue injected into the dorsolumbar epidural space in cows. Vet. Aneasth. Analg., v.28, p.140-145, 2001 .

LEE, I.; YAMAGISHI, N.; OBOSHI, K. et al. Effect of postural change on lumbar epidural pressure in cattle. Vet. J., v.164, p.292-294, 2002.

MERRY, A.F.; CROSS, J.A.; MAYADEO, S.V.; WILD, C.J. Posture and the spread of extradural analgesia in labour. Br. J. Aneasth., v.85, p.303306, 1983.

NAGANOBU, K.; HAGIO, M. The effect of body position on the 'hanging drop' method for identifying the extradural space in anaesthetized dogs. Vet. Aneasth. Analg., v.54, p.59-62, 2007.

NESS T.J.; GEBHART G.F. Visceral pain: a review of experimental studies. Pain, v.41, p.167234, 1990.

NYSTROM, E.U.M.; BLOMBERG, S.G.; BUFFINGTON, C.W. Transmural pressure of epidural veins in the thoracic and lumbar spine of pigs. Anesthesiology, v.89, p.449-455, 1998.

ROCHA, L.M.S.; MASSONE, F.; FREIRE, C.D. et al. Estudo anatomo-anestesiológico do segmento lombar (L1 a L6) em cães. Braz. J. Vet. Res. Anim. Sci., v.43, p.167-177, 1990.

SALMENPERA, M.T.; SZLAM, F.; HUG, C.C.J. Anesthetic and hemodynamic interactions of dexmedetomidine and fentanyl in dogs. Anesthesiology, v.80, p.837-846, 1994.
SEN, O.; FERAH DONMEZ, N.; ORNEK, D. et al. Effects of epidural needle rotation and different speeds of injection on the distribution of epidural block. Rev. Bras. Anestesiol., v.62 p.852862, 2012.

SON, W.; KIM, J.; SEO, J. et al. Cranial epidural spread of contrast medium and new methylene blue dye in sternally recumbent anaesthetized dogs. Vet. Aneasth. Analg., v.38, p.510-515, 2011.

SON, W.G.; JANG, M.; YOON, J.; LEE, L.Y.; LEE, I. The effect of epidural injection speed on epidural pressure and distribution of solution in anesthetized dogs. Vet. Aneasth. Analg., v.41, p.526-533, 2014.

STEAGALL, P.V.M.; SIMON, B.T.; TEIXEIRA NETO, F.J.; LUNA S.P.L. An update on drugs used for lumbosacral epidural anesthesia and analgesia in dogs. Front.Vet. Sci., v.4, p.1-11, 2017.

STEAGALL, P.V.M.; TEIXEIRA NETO, F.J.; MINTO, B.W. et al. Evaluation of the isofluranesparing effects of lidocaine and fentanyl during surgery in dogs. J. Am. Vet. Med. Assoc., v.229 p.522-527, 2006.

VALVERDE, A. Epidural analgesia and anesthesia in dogs and cats. Vet. Clin. N. Am. Small Anim. Pract. v.38, p.1205-1230, 2008.

VISSER, W.A.; LEE, R.A.; GIELEN, M.J.M. factors affecting the distribution of neural blockade by local anesthetics in epidural anesthesia and a comparison of lumbar versus thoracic epidural anesthesia. Aneasth. Analg., v.107, p.708-721, 2008.

ZHANG, D.; FUJIWARA, R.; ISERI, T. et al. Distribution of contrast medium epidurally injected at thoracic and lumbar vertebral segments. J. Vet. Med. Sci., v.75, p.663-666, 2013. 